\title{
A Kind Of Improved Adaptive Control Method And Its Application In Parameter Identification
}

\author{
Jun Peng ${ }^{1, a}$, Di Liu ${ }^{2, b}$, Junwei Lei ${ }^{2, c}$ \\ ${ }^{1}$ Receiving and Training Center of New Equipments, Naval Aeronautical and Astronautical \\ University, Yantai, China \\ ${ }^{2}$ Department of control engineering, Naval aeronautical and astronautical University Yantai, China \\ apengjun1024@126.com, 'Teijunwei@126.com
}

Keywords: adaptive control, parameter identification, one order system

\begin{abstract}
In order to achieve adaptive control and single parameter identification of one order system, an ordinary adaptive control method is used in this paper. By improving the adaptive control method, an adaptive control law is designed, and the result of parameter identification is analysed. In the end, a conclusion can be made that improved adaptive control method can't ensure parameter be identified, and the error of system can't converge to zero by numerical simulation.
\end{abstract}

\section{Introduction}

Parameter identification is a main problem that is discussed in many control systems because it is a kind of simple uncertain problem that can be solved by adaptive control method ${ }^{[1-9]}$. As the demand for control performance increases, the problem of time-variance parameters of controlled system is more and more important. A lot of parameter identification method is proposed by specialists and representatives from China and abroad. The classical parameter identification method is least square method ${ }^{[1]}$, Kalman filtering method, adaptive. The method modern parameter identification method is neural network method ${ }^{[2]}$, genetic algorithm method ${ }^{[3]}$ and particle swarm optimization. But calculating amount of these method is large, instantaneity and astringency can't meet the demands of the control system. So an ordinary adaptive control method is improved in this paper.

\section{Problem Description}

One order system can be written as:

$$
\dot{x}=a x+u
$$

where $a$ is unknown constant parameter, the goal is designing a controller such that the system state $x$ can trace the expected value $x^{d}$.

\section{Design Improved Adaptive Identification Controller}

An ordinary adaptive control method is used as follows, define a error variable as $z_{1}=x_{1}-x_{1}^{d}$, then

$$
\dot{z}_{1}=\dot{x}_{1}-\dot{x}_{1}^{d}=a x+u
$$

Design state feedback control law as:

$$
u=-\hat{a} x-\sum_{i=1}^{n} k_{i} f_{i}\left(z_{1}\right)
$$

Choose $n=1, k_{i}>0, f_{1}\left(z_{1}\right)=z_{1}$. design regulating law:

$$
\dot{\hat{a}}=\Gamma_{a} z_{1} x-k_{a d 1} \hat{a}
$$

Define $\tilde{a}=a-\hat{a}$, then: 


$$
\dot{\tilde{a}}=-\dot{\hat{a}}=-\Gamma_{a} Z_{1} x+k_{a d 1} \hat{a}=-\Gamma_{a} Z_{1} x+k_{a d 1} a-k_{a d 1} \tilde{a}
$$

Then there is:

$$
\begin{aligned}
& \dot{z}_{1}=\tilde{a} x-k_{1} z_{1} \\
& \dot{\tilde{a}}=-\Gamma_{a} z_{1} x-k_{a d 1} \tilde{a}+k_{a d 1} a
\end{aligned}
$$

choose Lyapunov function:

$$
V=\frac{1}{2} z_{1}^{2}+\frac{1}{2 \Gamma_{a}} \tilde{a}^{2}
$$

Then:

$$
\begin{aligned}
& \dot{V}=\tilde{a} x z_{1}-k_{1} z_{1}^{2}+\frac{1}{\Gamma_{a}} \tilde{a} \dot{\tilde{a}} \\
& =\tilde{a} x z_{1}-k_{1} z_{1}^{2}+\frac{1}{\Gamma_{a}} \tilde{a}\left(-\Gamma_{a} z_{1} x-k_{a d 1} \tilde{a}+k_{a d 1} a\right) \\
& =-k_{1} z_{1}^{2}-\frac{k_{a d 1}}{\Gamma_{a}} \tilde{a}^{2}+\frac{k_{a d 1}}{\Gamma_{a}} \tilde{a} a
\end{aligned}
$$

Obviously,there is

$$
\frac{k_{a d 1}}{\Gamma_{a}} \tilde{a} a \leq \frac{k_{a d 1}}{2 \Gamma_{a}}\left(\tilde{a}^{2}+a^{2}\right)
$$

Then:

$$
\begin{gathered}
\dot{V} \leq-k_{1} z_{1}^{2}-\frac{k_{a d 1}}{\Gamma_{a}} \tilde{a}^{2} \\
\leq-k_{1} z_{1}^{2}-\frac{k_{a d 1}}{2 \Gamma_{a}} \tilde{a}^{2}+\frac{k_{a d 1}}{2 \Gamma_{a}} a^{2} \\
\varepsilon_{v}=\min \left(2 k_{1}, \frac{k_{a d 1}}{\Gamma_{a}}\right) \quad \text { to meet } \\
\dot{V} \leq-\varepsilon_{v} V+\frac{k_{a d 1}}{2 \Gamma_{a}} a^{2}
\end{gathered}
$$

The system is stable, but the signal must be bounded, $\tilde{a}$ and $z_{1}$ is not zero. The control system that is based on improved integral adaptive control can't ensure parameter be identified, and the error of system can’t converge to zero.

\section{Numerical Simulation}

Choose unknown parameter $a=3$, initial state $x_{1}(0)=-1$, expected state $x_{1}^{d}=1$, use Simulink in Matlab, write program without improved integral adaptive identification controller, the program can be written as: 


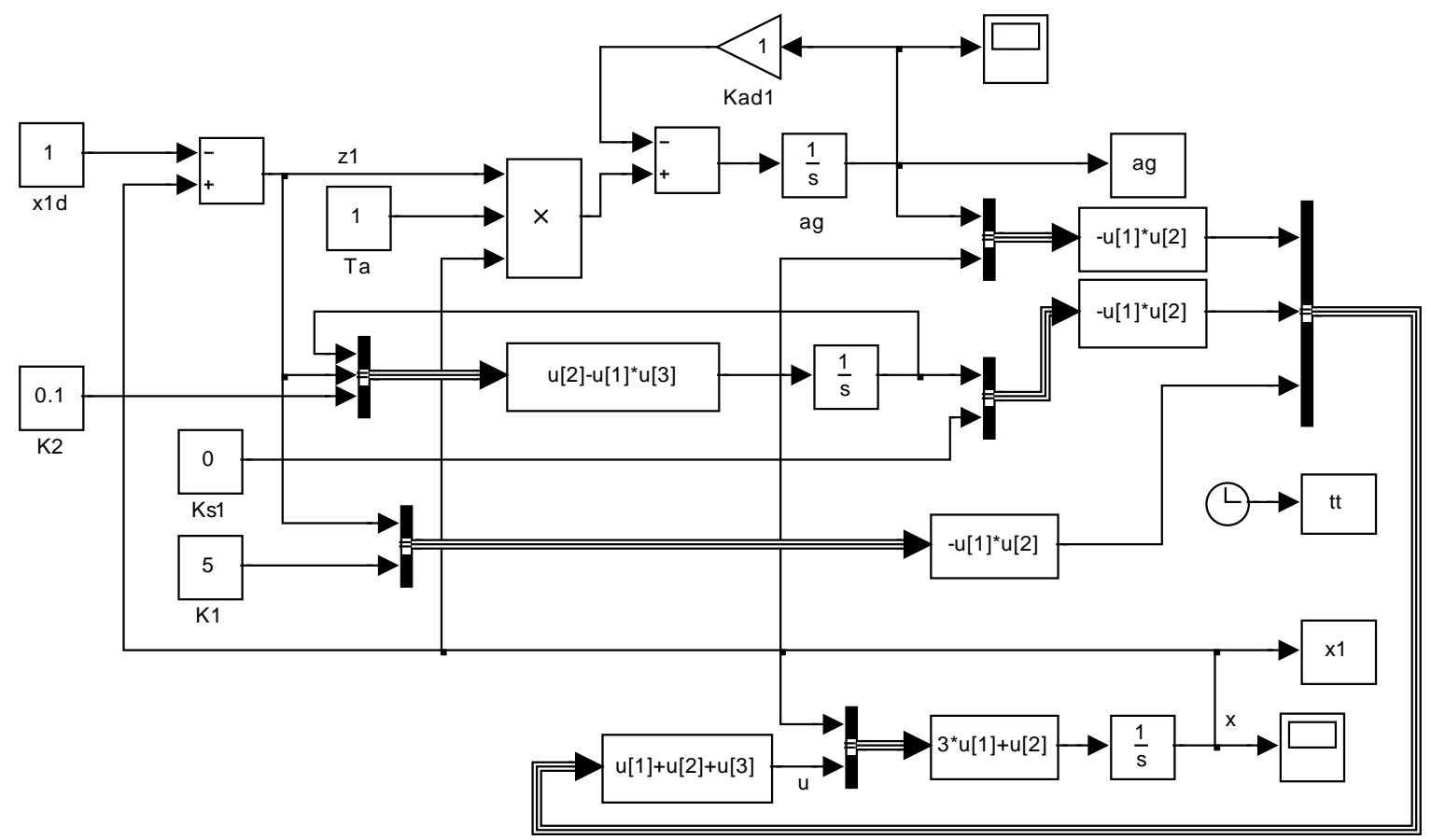

Fig.1 Program without improved integral

Choose $k_{1}=5, k_{s 1}=0, \Gamma_{a}=1, k_{a d 1}=1$, the simulation results are as follows:

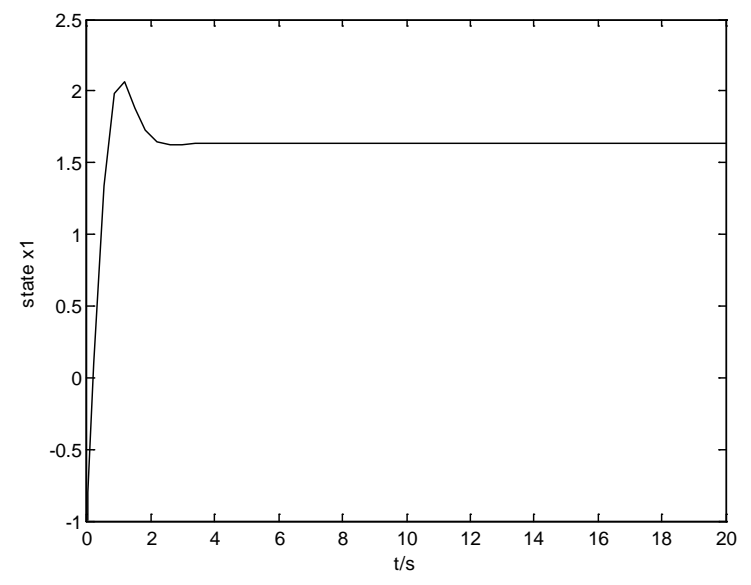

Fig.2 state a

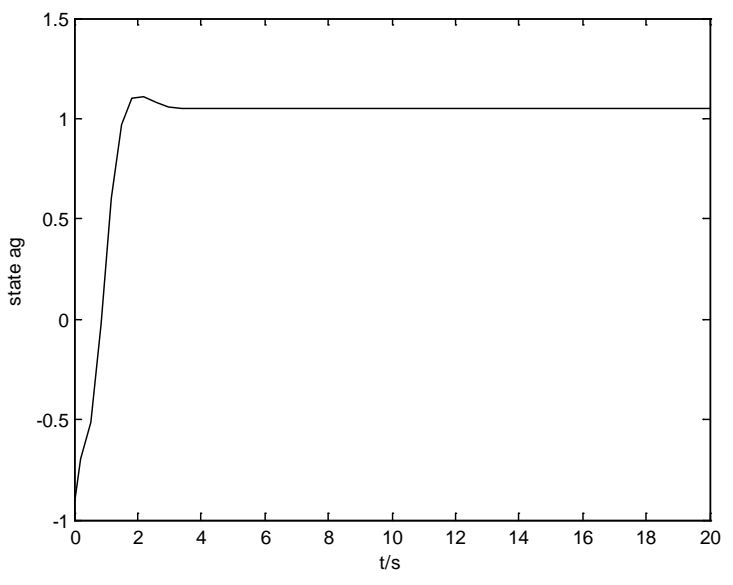

Fig.3 state $\mathrm{x} 1$

From the result, the error of system is large, and parameter can't be identified. The parameter can be identified on condition that there is no steady state error. When control gain is increased, the error decrease. Choose $k_{1}=500, k_{s 1}=0, \Gamma_{a}=1, k_{a d 1}=1$, the simulation results are as follows: 


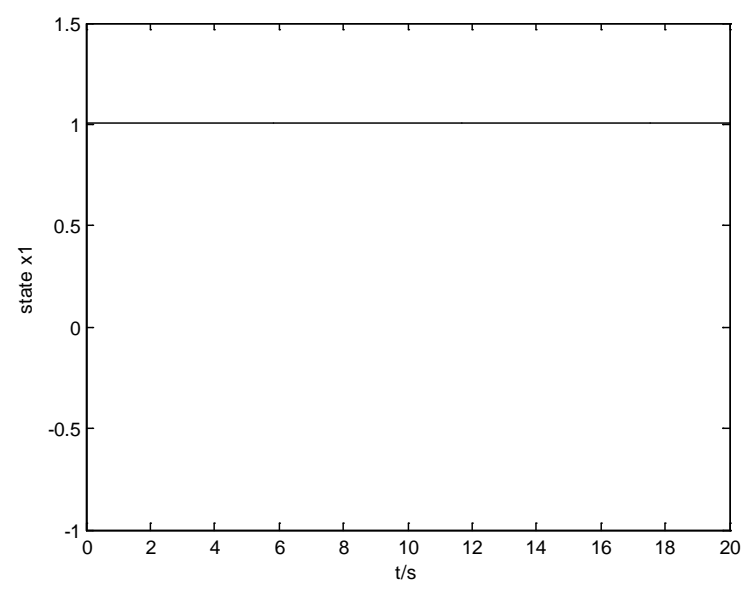

Fig. 4 state a

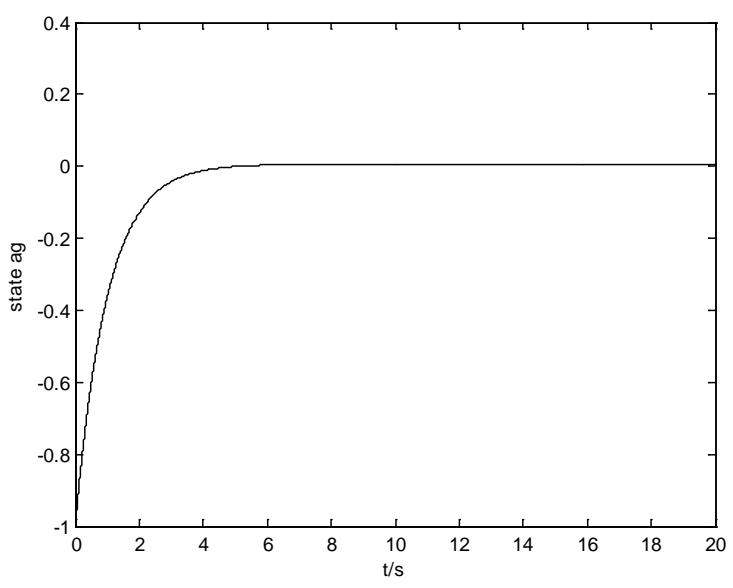

Fig. 5 state $\mathrm{x} 1$

The estimated parameter value converge to zero, and the error of system decrease. Choose $k_{1}=50, k_{s 1}=0, \Gamma_{a}=1, k_{a d 1}=1$, the simulation results are as follows:

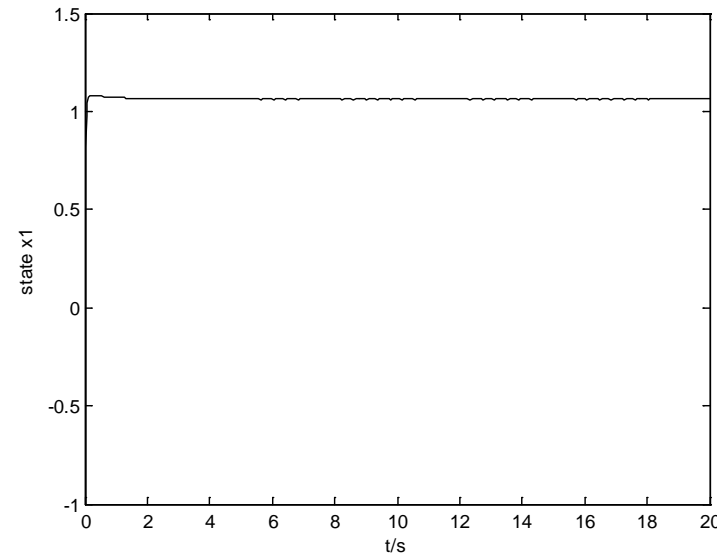

Fig.6 state a

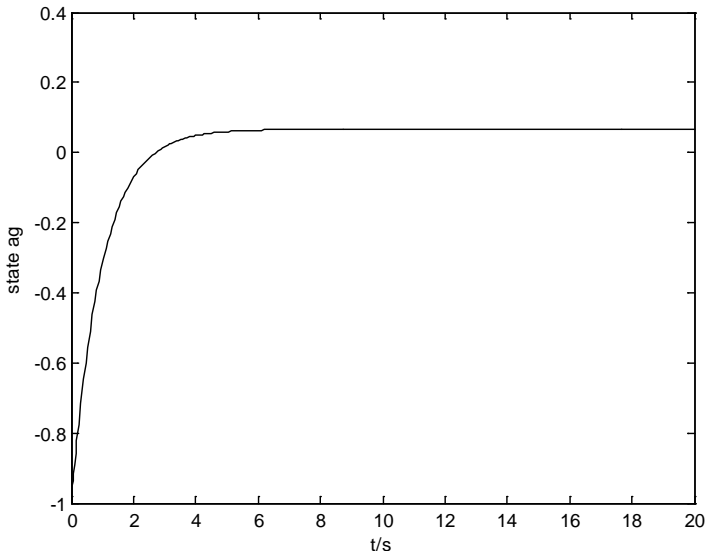

Fig.7 state $\mathrm{x} 1$

From the result, the unknown estimated parameter value is around the zero. The larger gain is, the smaller steady state error is, and the closer estimated parameter value is to zero.

\section{Conclusion}

According to the problem that system parameter need to be identified, an improved adaptive control is proposed in this paper. By analysing theory and the simulation results, we can make a conclusion that the improved method is not effective to parameter identification. The larger gain is, the smaller steady state error is, and the closer estimated parameter value is to zero, the parameter identification can;t be achieve.

\section{Reference}

[1] Maurizio C, Marcello P, Giansalvo, et al. A new experimental application of least-squares techniques for the estimation of the induction motor parameters[J]. IEEE Transactions on Industry Applications, 2003, 39(5):1247-1256.

[2] Raj M B, Alexander G P, Hamid A T, Neural speed filtering for sensorless induction motor drives[J]. Control Engineering Practice, 2004, 12(6): 687-706.

[3] Huang K S, Kent W, Wu Q, et al. Parameter identification of an induction machine using genetic algorithms[C] // Proceedings of the 1999 IEEE International Symposium on Computer Aided Control System Design. [S. L.]: [s. N.],1999: 510-515. 
[4] Huynh D C, Dunnugan M W. Parameter estimation of an induction machine using advanced particle swarm optimisation algorithms[J]. IET Electric Power Applications, 2010, 4(9): 748-760.

[5] C.J.Atkinson,R.G.Urso.Modeling of Apparent Mass Effects for the Real-Time Simulation of a Hybird Airship.AIAA Modeling and Simulation Technologies Conference and Exhibit,Keystone.2006:21 32

[6] Yokomaku Y.The Stratospheric Platform Airship R\&D Program of Japan. The 2nd Stratospheric Platform Systems Workshop,Tokyo Japan.2000:7 13

[7] S.P.Jones,J.D.Laurier.Aerodynamic Estimation Techniques for Aerostats and Airships.AIAA Lighter-than-Air Systems Conference,Annapolis,2004: 88 94

[8] M.T.Soylemeza,N.Munrob,H.Bakic.Fast Calculation of Stabilizing PID Controllers. Automatic, 2003,39(7):121 126

[9] Etkin B, Theory of the flight of Airplanes in Isotropic Turbulence Review an Extension,AGARD Rept.1961:372 